\section{How virtual reality is helping to rebuild a community devastated by the Fukushima disaster}

The Great East Japan Earthquake of 2011, and the accompanying tsunami that led to the meltdown presushima's nuclear pow powerful the world has see powerful the world has seer people. Thousands more were displaced and still don't know if they will ever return to town like Namie which they once called home. A new project led by Dr Kanako Sasaki from Tohoku University hopes Namie and is reconstructing its community online.

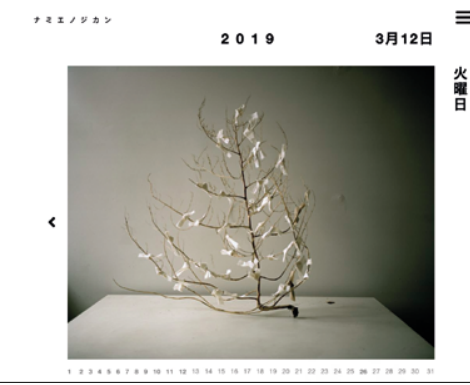

in recent years. Even before the disaster Japan was facing the challenge of how to cope with both an ageing society fewer eldely people live (as traditionally) with their eldest son in a multi-

As a result, although some Fukushima evacuees have gone to live with family members, many others, especially elderly feel - not only through being separated through their displacement from the fom a hir displacem that for generations formed the backdrop to their family's lives - is of increasing concen.

Also worrying is that, upon relocation, younger generations hesitate to talk when stating a cam for , for examp evacueee can be stresful and may make people feel stigmatised, and may make to bullying, because others wrongly think that radiation can be caught like a virus. They may also suffer jealousy from neighbours, for example, if they Japanese society has changed much

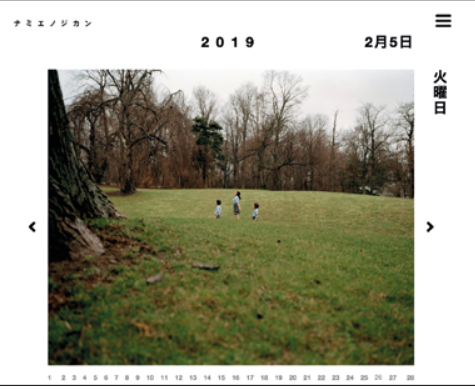
people, now live alone. The isolation they

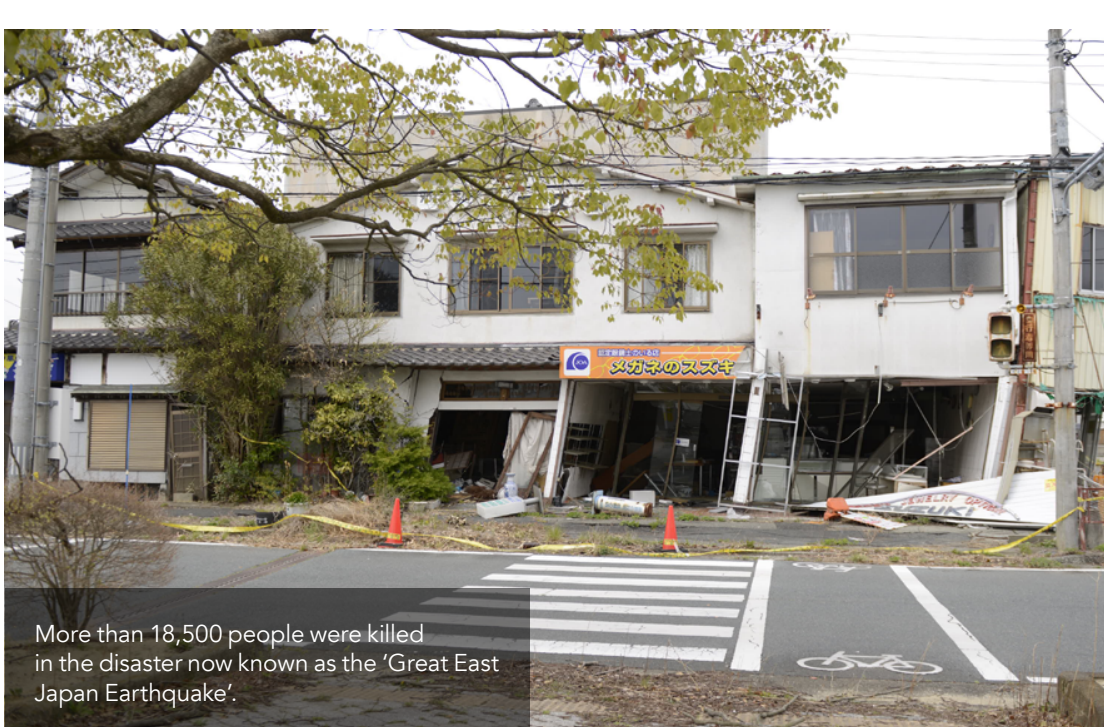

use their compensation money to build new house, or because they are entilit fes and highway tolls As a result many (2) and feel like they are living in hiding.

\section{NARRATIVE POWER}

Dr Kanako Sasaki from Tohoku University has been studying the effects of the inhabitants of one town in Fukushima province, Namie, the birthplace of her father. In 2010 Namie's population was around 22,000. Farming and fishing were the main sources of employment, but it was not a wealthy area and many family other parts of Japan.

The Tokyo Electric Power Company's (TEPCO) decision to build a nuclear ant nearby brought much-needed mployment. But one day after the evacuate, Namie's popultion fell to zero. Although some people began to return. when restrictions were lifted for some areas in 2017, many are reluctant to do so and fewer than $10 \%$ have so far returned.

With a background in the arts and sociology, Dr Sasaki's interest lies in the power of narrative - the told and untold stories of the disaster's survivors. For h project "Archive Namie: protecting memories", she began by interviewing Fukushima evacuees still living in explains: "Liconinodation. Dr Sasaki explains. "Listening to the compelling discovered that elderly person sunvivors

in particular had no choice but to accep in home of last resort."

The consequence is that many Fukushim survivors feel they can't talk about the disaster or where they have come from. It's as if the past has disappeared. In the wider context there is the added historica cost of people's memories and culture being lost to future generations.

Dr Sasaki aims to change this and help people tell their stories, anonymously they wish. She hopes that this wil the disaster to understand exverience and empathise with their situation. In addition, she hopes that turning evacuees' experiences into art will help them to release some of the feelings they have kept hidden. "This is the power of narrative - of live stories," she said.

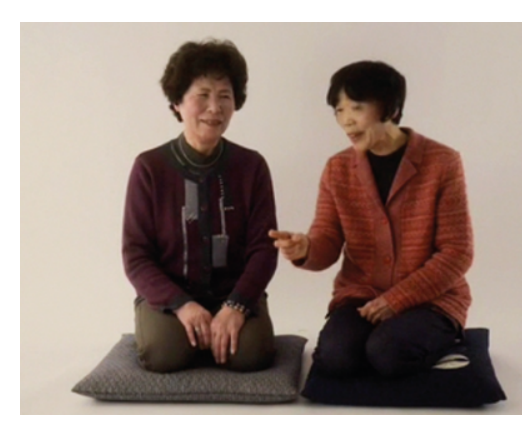

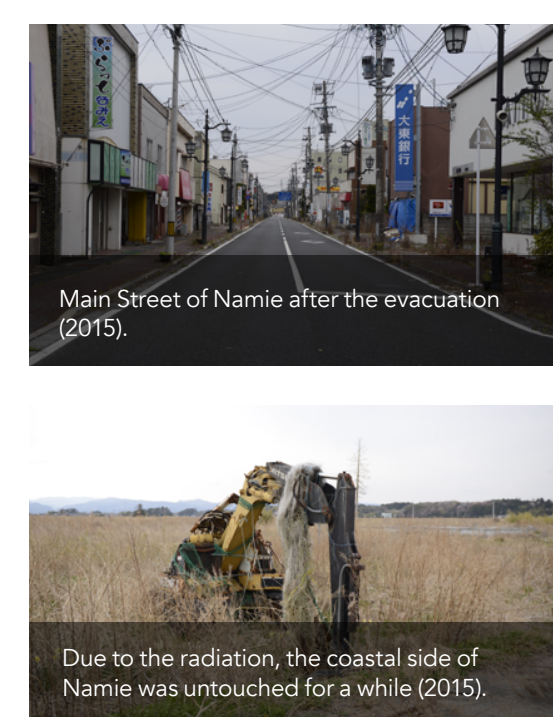

THE PROJECT

Dr Sasaki's project to archive people's memories of Namie is based online. anywhere they are now living and to communicate anonymously if they wish. By incorporating photography and

Accessing Namie via a phone line has a similar function to visiting a Shinto shrine.

as storytelling, for younger generations. Dr Sasaki explains: "This project aims to provide a place where (evacuees) can feel at home and securely able to express their own voice. It also aims to pass by introducing artistic expression.

\section{HOTLINE NAMIE}

There are three strands to the project so notline, a digital calenda Namie' links evacuees in a temporary housing centre with a programmed machine in Namie. People can listen

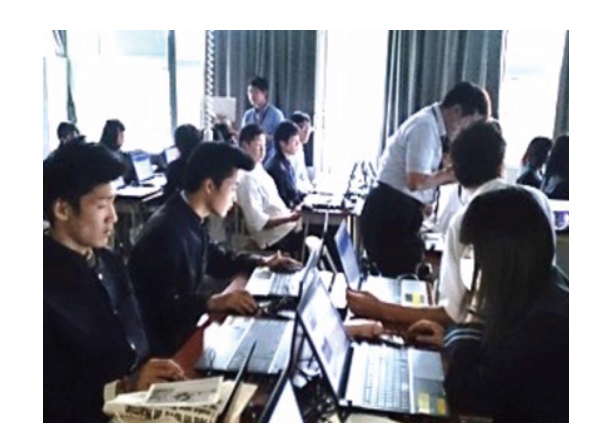

Left: The 'Archive Namie: protecting memories' project began by interviewing Fukushima evacues 
to sounds from Namie, for example of the sea, or speak to Namie by leaving ased the telephone and for some it a near-religious experience. As Dr Sasa says: "Accessing Namie via a phone line has a similar function as visiting a Shinto shrine to worship a deity and make a wish as in Japanese tradition. Having conversations with deceased people or sharing mundane experiences,

is a secretive and personal act."

One evacuee left the following message: "Dear deceased friends, can you hear me? What an inciden it was. I never thought such a thing would happen. Now, we are all isolated or stolen by the Tsunami.... Wishing we can vist our ancestors graves

\section{DIGITAL CALENDAR}

Dr Sasaki has also created an online people can upload photographs inspired by memories of their hom every day. As before, they can do this anonymously if they wish. Workshops were first held to help evacuees improve their photographic skills. They were also encouraged to think of their images as Haiku - traditional Japanese poems whic describe aspects of the physical world in a way that symbolises the poet's feelings.

People can upload text to explain why an image is meaningful for them perhaps because of the date or season. For example, one evacuee and explained how he used to halp neighbours: "We used horses to puddle the field. Weeding the field

was the tough one."

On other occasions people might upload text, for which Dr Sasaki has created accompanying images. For example, she uploaded the image of a broken plate to illustrate this moving testimony: "Sounds of something cracking at midnight. I figured out why as I grew up. It's hard to be a daughterThe third aspect of the project is 'Memorytalk', a social networking
platform that allows users to archive text,

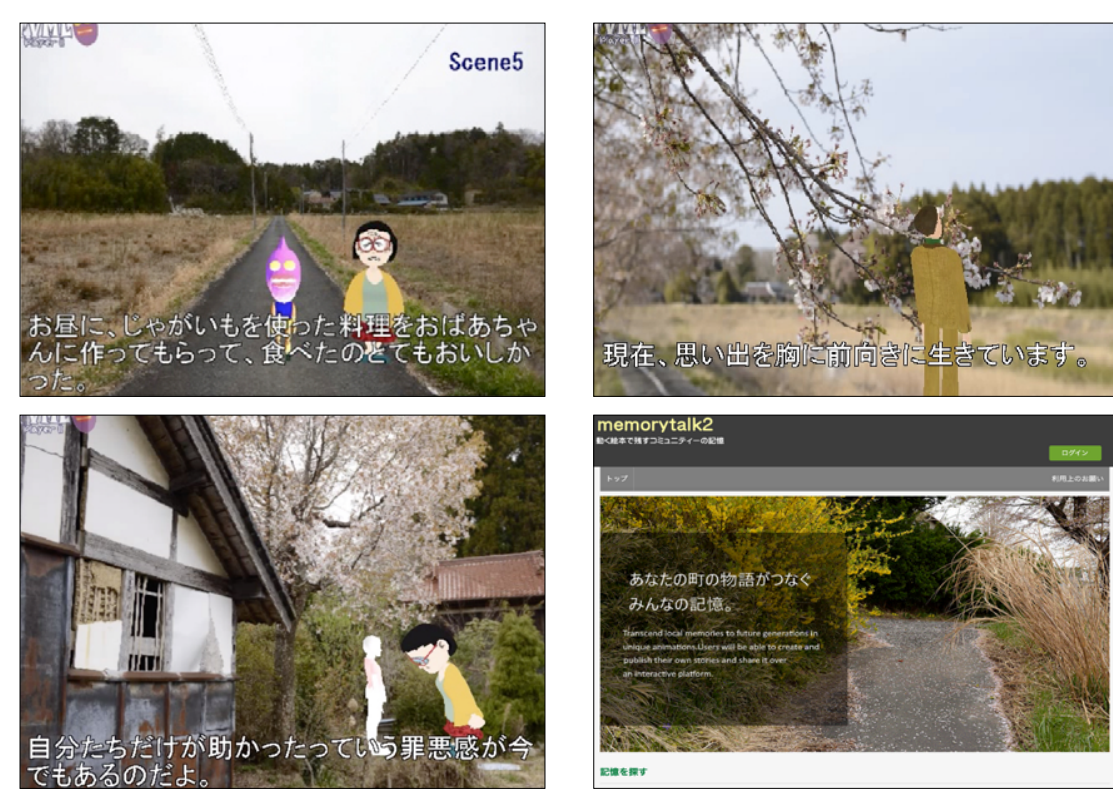

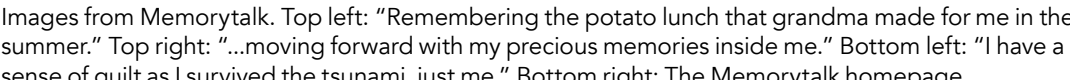

This project aims to provide a place where evacuees feel at home and are securely able to express their own voice.

videos and photographs about Namie. The aim is to create a virtual community which allows users to record their own
memories which is hoped will stimulate memories which is hoped will stimulate can also turn their stories into animations through the development of online tools.

Users first choose one of 84 characters or 'avatars' to be their online narrator. which allows them to be anonym They also choose a background image and soundtrack against which monologu or a diague with of avatars. They can make their avatars move by choosing gestures, for examplo jumping, bowing or smiling and when al inputs are selected, the animation plays at the touch of a button.

Dr Sasaki hopes the introduction of animation into storytelling will result in Memorytalk succeeding where other archive projects have failed, for example through being expensive to maintain or having declining user numbers. Othe sites have also been aimed at learning lessons from people's experience to help prevent similar disasters in the future. Unlike Memorytak, they have not been about helping evacuees to make sense
Dr Sasaki said: "Since Memorytalk is platform users can communicate with each oher online by commenting the uploaded content. This enerating various life stories. Keeping communication online might also bring users back to the site regularly, which can help to keep the archive alive."

\section{RECONNECTION}

Dr Sasaki's project is ongoing

and its success has yet to be reviewed.

However, the quality of communication in the new virtual community is

impressive and an archive is being built to help future generations know more about Namie's past. Above all Dr Sasak hopes her work will not only help evacuees with their hometown, but that it will also help people get in touch with their inner selves.

Dr Sasaki says: "Evacuees are relocated all over Japan, yet this project hopes to bring them home virtually by sharing and participating. The of daily life and he prestés memories of daily life and help to preserve local culture, helping people to remember an SNS-based (social network system)

12

\section{Behind the Research}

Dr Kanako Sasaki

E: kanarinus0919@gmail.com T: +81-90-4042-3179 W: kanakosasaki.com

\section{Research Objectives}

Dr Kanako Sasaki's research is focused on media study under art and sociology, in particular exploring the power of narrative.

\section{Detail}

Dr Kanako Sasaki

Tohoku University Graduate school of Information Science Aoba 6-3-09,

aba-ku Sendai-city Miyagi-pref.

980-8579, Japan

Bio

Kanako Sasaki is a post doctoral fellow at Tohoku University, Japan. She is a founder of Archive Namie, experimenting and challenging the way of archiving memories in various in photography.

Funding

JSPS KAKENHII Grant Number JP 18K18326

- Foundation of Fusion of Science and Technology

Collaborators

People from the town of Namie

- Oohori lab, Tohoku University

echnology

- Design team

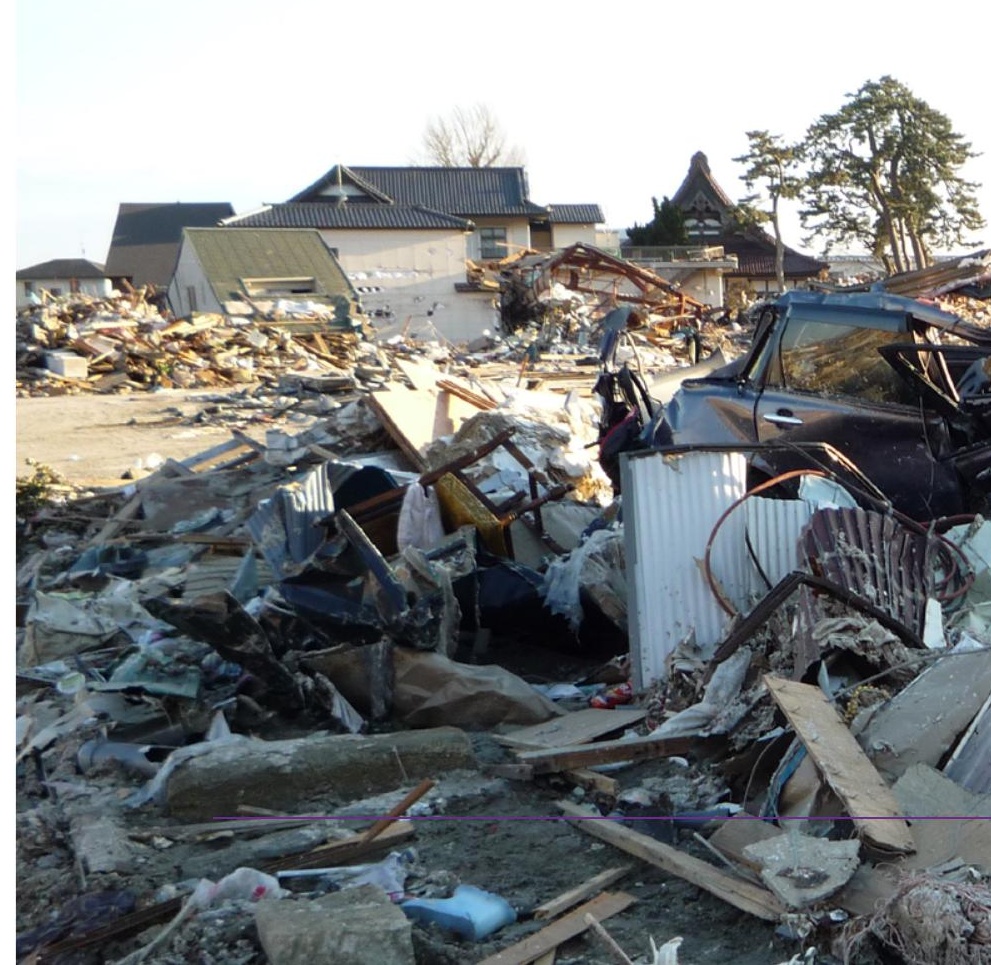

\section{References}

Sasaki, K. and Sakurai. M. (2015). 'Voluntary Isolation After the Disaster: The Loss of Community and Family in the Super Aged Society in Japan.' Journal of Disaster Research, Vol 10,

\section{Personal Response}

Many of the elderly Fukushima survivors may not be used to the digital online world that younger people now take for granted. How have they reacted to going
online and, for example, using avatars and animations online and, for examp
to tell their stories?

II It is true that elderly survivors have difficulty using IT. this is why created the Hotline Namie, which allowed different media for different target groups. For example. I tried the Memorytalk, the animation app, to target teenagers. Memorytalk involves a lot of steps to upload the entries. It is not user-friendly for elderly people. However, the town of Namie distributed tablet devices, communicate with distant families. Elderly people are gradually getting used to IT devices and the digital. calendar was very easy for even them to use.

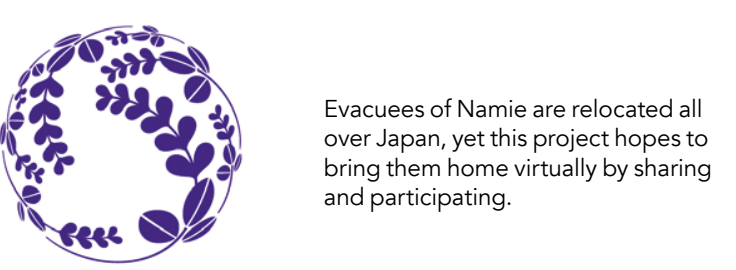

TOHOKU 